\title{
Hybrid entanglement for quantum information and communication applications
}

Nape, I., Ndagano, B., Perez-Garcia, B., HernandezAranda, R., Roux, F., et al.

I. Nape, B. Ndagano, B. Perez-Garcia, R. I. Hernandez-Aranda, F. S. Roux, T. Konrad, A. Forbes, "Hybrid entanglement for quantum information and communication applications," Proc. SPIE 10347, Optical Trapping and Optical Micromanipulation XIV, 1034711 (25 August 2017); doi: 10.1117/12.2274361

Event: SPIE Nanoscience + Engineering, 2017, San Diego, California, United States 


\title{
Hybrid entanglement for quantum information and communication applications
}

\author{
Nape. I ${ }^{a}$, Ndagano B. ${ }^{a}$, Perez-Garcia B. ${ }^{\mathrm{a}, \mathrm{b}}$, Hernandez-Aranda R.I. ${ }^{\mathrm{b}}$, Roux F.S. ${ }^{\mathrm{c}}$, Konrad T \\ d, and Forbes A. ${ }^{\text {a }}$ \\ ${ }^{a}$ University of the Witwatersrand, 1 Jan Smuts Avenue, Johannesburg, South Africa \\ bPhotonics and Mathematical Optics Group, Tecnológico de Monterrey, Monterrey 64849, \\ Mexico \\ ${ }^{\mathrm{c}}$ National Metrology Institute of South Africa, Meiring Naude Road, Pretoria, South Africa \\ ${ }^{\mathrm{d} C o l l e g e}$ of Chemistry and Physics, University of KwaZulu-Natal, Private Bag X54001, \\ Durban 4000, South Africa
}

\begin{abstract}
Combining the multiple degrees of freedom of photons has become topical in quantum communication and information processes. This provides advantages such as increasing the amount of information that is be packed into a photon or probing the wave-particle nature of light through path-polarisation entanglement. Here we present two experiments that show the advantages of using hybrid entanglement between orbital angular moment (OAM) and polarisation. Firstly, we present results where high dimensional quantum key distribution is demonstrated with spatial modes that have non-separable polarisation-OAM DOF called vector modes. Secondly, we show that through OAM-polarisation entanglement, the traditional which-way experiment can be performed without using the traditional physical path interference approach.
\end{abstract}

Keywords: hybrid entanglement, quantum erser, quantum key distribution, orbital angular momentum

\section{INTRODUCTION}

Entangled photons have become a ubiquitous source for quantum communication and information. Various degrees of freedom (DoF) have been exploited in entangled systems, e.g., polarization, ${ }^{1,2,3}$ time, ${ }^{4,5,6}$ spatial modes of orbital angular momentum ${ }^{7,8,9,10}$ and transverse linear momentum. ${ }^{11}$ However, combing multiple DoF has shown significant promise. For example, the increasing requirements for transmitting large amounts of information in quantum systems can be met by combining multiple DoF in so called hyper entangled systems. ${ }^{12}$ Interestingly, even the fundamental features of quantum systems have been exploited through hybrid entangled quantum systems ${ }^{13}$ wherein two systems exhibit entanglement in differing DoF. For example, the polarization of one photon can be manipulated to demonstrate the erasure of the path information of a distant photon.

Hybrid entanglement also manifests itself in single photon states; the DoF of the photons are non-separable although non-locality does not hold. Interestingly, polarization and OAM DoF can exhibit this form of entanglement in spatial modes called vector vortex modes (or vector modes). The contribution of high dimensional OAM and qubit polarization DoF allows for larger alphabets to be exploited. ${ }^{14}$ They have featured in applications ranging from quantum error correction schemes ${ }^{15}$ and high bandwidth classical optical communication. ${ }^{16}$ In quantum cryptography, these hybrid photon states have only been exploited for their rotation invariance. ${ }^{17,18}$

Here we exploit hybrid entanglement to demonstrate the advantage of using quantum systems with combined DoF. First, in Sec. 2.1 we present a quantum eraser scheme that does not involve physical path interference. We achieve this by replacing the concept of paths with twisted photon states (OAM). Just as in the traditional quantum eraser experiment, we show that the OAM information of a photon can be erased. Secondly, in Sec. 2.2, we employ these non-separable quantum states, reminiscent of polarization-OAM hybrid entanglement (vector modes) ${ }^{19,20}$ to demonstrate a high dimensional quantum key distribution scheme.

Further author information: E-mail: isaacnape@gmail.com

Optical Trapping and Optical Micromanipulation XIV, edited by Kishan Dholakia, Gabriel C. Spalding,

Proc. of SPIE Vol. 10347, 1034711 - (c) 2017 SPIE · CCC code: 0277-786X/17/\$18 · doi: 10.1117/12.2274361 
(a)

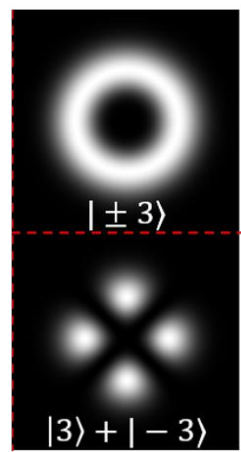

(b)



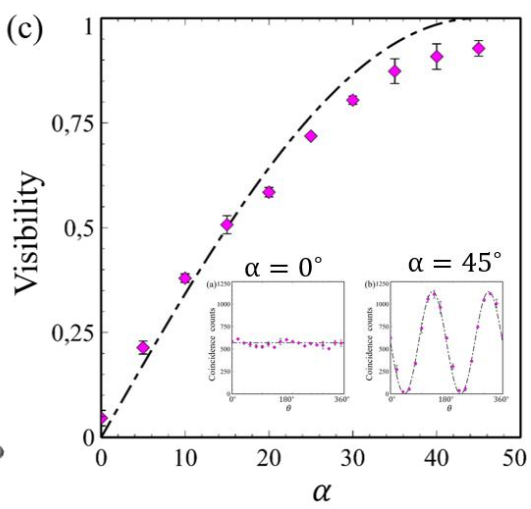

Figure 1. (a) Example of intensity profiles for $\ell= \pm 3$ modes and their superposition. Experimental set-up used to perform the OAM entanglement quantum eraser. (c) Experimental results for the spatial fringe visibility measurements. The inserts depict the extreme cases; when there is OAM information $\left(\alpha^{\circ}=0\right)$ and once it is erased $\left(\alpha=45^{\circ}\right)$ from the system. The two cases are complimentary ${ }^{21}$

\section{RESULTS}

\subsection{Quantum eraser using hybrid entanglement}

A quantum eraser can be engineered from hybrid entanglement, enabling the information of quantum system to be erased by exploiting the particle and wave nature of photons. ${ }^{13}$ For example, in the double slit quantum eraser, the paths of a distant photon can be marked with the polarization of another. The state of the system is given by

$$
|\Phi\rangle=\frac{1}{\sqrt{2}}(|H\rangle|a\rangle+|V\rangle|b\rangle),
$$

with $|a\rangle$ and $|b\rangle$ being the photon states upon traversing the independent paths $a$ and $b$, and $|H\rangle$ and $|V\rangle$ represent the horizontal and vertical polarization states that mark the two paths, respectively. Remarkably, the path DoF can be treated as two level system on the Hilbert space which can be described equivalently for any DoF. We demonstrate this with OAM.

OAM can be described on a 2 dimensional subspace $\mathcal{H}_{\ell}=\operatorname{span}\{|\ell\rangle,|-\ell\rangle\}$ where $\pm \ell$ is the topological charge with the sign determining the handedness of azimuthal phase rotations due to angular momentum ${ }^{22}$ ). Remarkably, OAM eigenmodes have uniform azimuthal intensity pattern and their superpositions have azimuthal spatial fringes (see Fig. 1(a)) for an example with $\ell= \pm 3$ ).

To demonstrate a quantum eraser with OAM we used polarisation-OAM hybrid entanglement engineered from spontaneous parametric down conversion (SPDC) and geometric phase control. ${ }^{23}$

$$
|\psi\rangle_{s, i}=\frac{1}{\sqrt{2}}\left(|H\rangle_{s}|\ell\rangle_{i}+i|V\rangle_{s}|-\ell\rangle_{i}\right) .
$$

Here Eq. (2) represents a system with the OAM abstract paths of the idler (i) photon marked with orthogonal $|H\rangle$ and $|V\rangle$ linear polarization states of the signal (s) photon. Significantly, Eq. (2) means that marking the OAM modes (projecting the signal onto $|H\rangle$ or $|V\rangle$ ) of the idler photon induces a uniform azimuthal intensity pattern and erases the OAM information (projecting the signal onto $\frac{1}{\sqrt{2}}(|H\rangle \pm|V\rangle$ ) causes the formation of azimuthal spatial fringes due to a collapse into a superposition state of OAM $\left(\frac{1}{\sqrt{2}}(|\ell\rangle \pm|-\ell\rangle)\right)$.

Our experimental set-up is illustrated in 1(b). We produced OAM entangled photons and engineered an orbit to spin conversion using a $q$-plate ${ }^{23,24}$ in the signal photon arm and used a quarter wave plate to convert from circular to linear polarization. A polarizer oriented at $\alpha$ was inserted before the avalanche photon detector in the idler arm and rotated from $0^{\circ}$ to $45^{\circ}$, acting to obtain and erase OAM information. A spatial light modular 
(SLM) encoded with a phase step was used to scan the spatial structure of the idler photon. The phase step projects onto the state $|\theta\rangle=\frac{1}{\sqrt{2}}\left(|\ell\rangle+e^{2 \theta}|-\ell\rangle\right)$. By rotating the hologram, we could scan the azimuthal spatial distribution of the photons. This is a technique used in Bell-like inequality measurements. ${ }^{10}$ We present the experimental visibility measurement results in Fig. 1(c).

At $\alpha=45^{\circ}$, i.e. selecting the $|H\rangle$ state, we measured spatial fringes with visibilities of $V=0.04 \pm 0.01$ in the idler arm which indicates that the idler photon had a well defined OAM state, analogous to marking the physical paths of a double slit and observing no interference fringes (particle-like behavior). For $\alpha=45^{\circ}$, corresponding to projecting onto the state $\frac{1}{\sqrt{2}}(|H\rangle+|V\rangle)$ we measured a fringe visibility of $V=0.92 \pm 0.01$, indicating that the OAM information had been erased, reminiscent to erasing path information in the double slit experiment. ${ }^{25}$

\subsection{Quantum communication with vector modes}
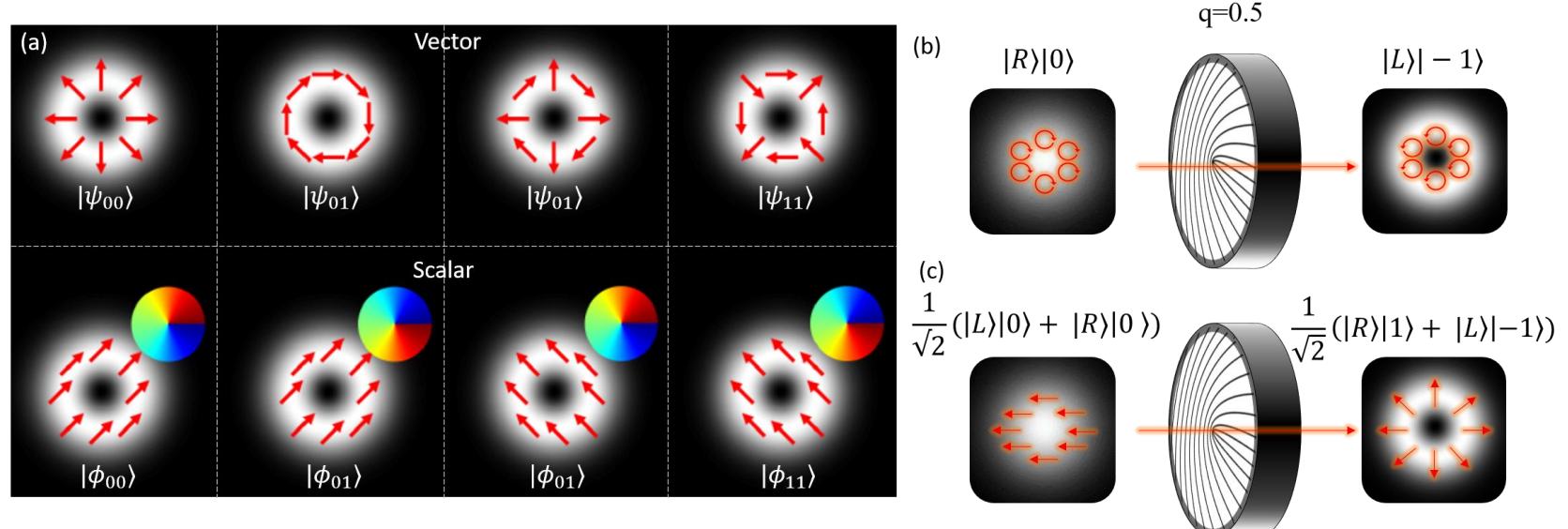

Figure 2. (a)The intensity and polarization field profiles for the vector and scalar modes for $|\ell|=1$. The inserts represent azimuthal phase profiles. (b) Typical generation scheme for scalar and vector modes using a $q$-plate.

Here we demonstrate a high dimensional BB84 protocol with spatial modes that are coupled in polarization and OAM DoFs. These spatial modes span the high dimensional Hilbert space formed by the tensor product between the $\mathrm{OAM}\left(\mathcal{H}_{\ell}=\operatorname{span}(\{|\ell\rangle,|\ell\rangle\})\right)$ and polarization $\left(\mathcal{H}_{\sigma}=\operatorname{span}(\{|R\rangle,|L\rangle\})\right)$ state spaces, i.e. $\mathcal{H}_{\sigma, \ell}=$ $\mathcal{H}_{\sigma} \otimes \mathcal{H}_{\ell}$, called the higher order Poincareé sphere. ${ }^{14}$ As a result, a four dimensional basis can be constructed from $\mathcal{H}_{\sigma, \ell}$, i.e. $\{|\ell, L\rangle,|-\ell, L\rangle,|\ell, R\rangle,|-\ell, R\rangle\}$. Using $\mathcal{H}_{\sigma, \ell}$, we generate a four dimensional basis comprised of superpositions of hybrid photon states called vector modes which we will use as our standard encoding basis:

$$
\begin{aligned}
\left|\psi_{00}\right\rangle & =\frac{1}{\sqrt{2}}(|R\rangle|\ell\rangle+|L\rangle|-\ell\rangle) \\
\left|\psi_{00}\right\rangle & =\frac{1}{\sqrt{2}}(|R\rangle|\ell\rangle-|L\rangle|-\ell\rangle) \\
\left|\psi_{10}\right\rangle & =\frac{1}{\sqrt{2}}(|R\rangle|-\ell\rangle+|L\rangle|\ell\rangle) \\
\left|\psi_{11}\right\rangle & =\frac{1}{\sqrt{2}}(|R\rangle|-\ell\rangle-|L\rangle|\ell\rangle) .
\end{aligned}
$$

The spatial modes described by $\left|\psi_{k j}\right\rangle$ exhibit polarisation-OAM coupling in a non-separable fashion, reminiscent 
of hybrid entanglement. We also selected a set of mutual unbiased of scalar modes,

$$
\begin{aligned}
\left|\phi_{00}\right\rangle & =|D\rangle|\ell\rangle \\
\left|\phi_{01}\right\rangle & =|D\rangle|-\ell\rangle \\
\left|\phi_{10}\right\rangle & =|A\rangle|\ell\rangle \\
\left|\phi_{11}\right\rangle & =|A\rangle|-\ell\rangle,
\end{aligned}
$$

such that $\left|\langle\psi \mid \phi\rangle^{2}\right|=\frac{1}{4}$. Our scalar modes have diagonal $(|D\rangle)$ and anti-diagonal $(|A\rangle)$ polarizations with OAM modes with $\pm \ell$ (see Fig. 2 (c) for the intensity and field profiles). The vector modes $\left(\left|\psi_{k j}\right\rangle\right)$ have spatially varying polarizations due to their non-separability while, on the contrary, scalar modes $\left(\left|\phi_{k j}\right\rangle\right)$ have homogeneous states of polarizations.

We used a attenuated laser source with a wavelength of $\lambda=633 \mathrm{~nm}$ and an average photon number of near $\mu=0.1$ photons per pulse. We used $q$-plates to generate the spatial modes sets through geometric phase and polarization control guided by the following rules polarization

$$
\begin{aligned}
& |L\rangle|\ell\rangle \stackrel{q \text {-plate }}{\longrightarrow}|L\rangle|\ell+2 q\rangle, \\
& |R\rangle|\ell\rangle \stackrel{q \text {-plate }}{\longrightarrow}|L\rangle|\ell-2 q\rangle .
\end{aligned}
$$

Here $q$ is the topological charge of the $q$-plate where 0.5 was chosen. We generated the scalar modes by transforming an input circularly polarized Gaussian mode with wave plates and $q=1 / 2$ - plates. For example, we generated the modes $\left|\phi_{00}\right\rangle$ by preparing a right circularly polarized Gaussian beam $(|L, 0\rangle)$. Applying the $q$ plate we obtained $|L, 0\rangle \rightarrow|R, 1\rangle$. The diagonal polarisation state is obtained through polarisation control using wave plates. The preparation of vector modes requires the input polarization (at the $q$-plate) thus resulting in non-separable superposition states of $|\psi\rangle_{k j}$.
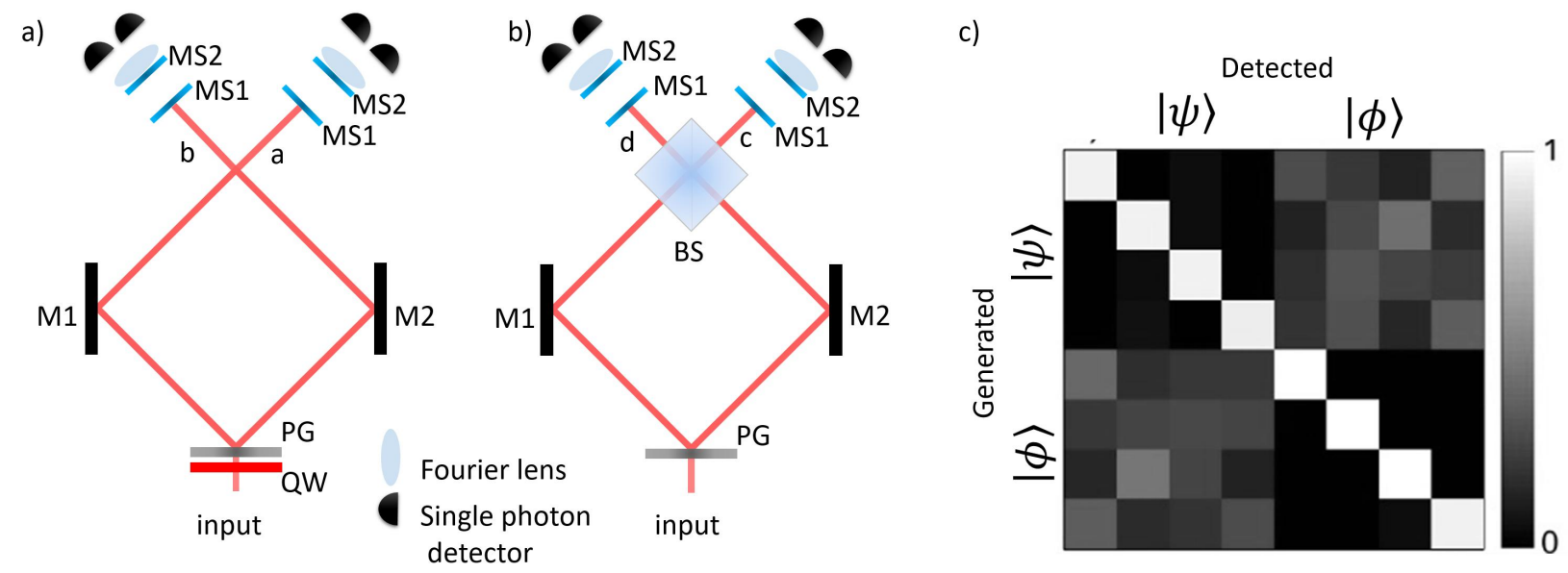

Figure 3. Detection scheme for sorting the (a) scalar and (b) vector mode sets. The scalar modes are first converted from linear to circular polarization with quarter a quarter wave (QW) plate then subsequently resolved into arms a and b by the polarization grating (PG) and mapped onto unique positions using OAM modes sorter elements MS1 and MS2. The vector modes are mapped onto paths $\mathrm{c}$ and d after the 50/50 beam-splitter (BS) and then mapped onto unique positions with the MS1 and MS2. (c) The measured probability scattering matrix obtained from preparing and measuring in the $\ell= \pm 1$ subspace.

We detected the mode sets with a deterministic scheme that probes the $\mathcal{H}_{\sigma, \ell}$ subspace with unit probability. Each mode was mapped onto a unique position using OAM mode sorters. ${ }^{26}$ The mode sorters are refractive optical elements (MS1 and MS2) that map OAM to position after a Fourier lens. ${ }^{26,27,28,29}$ Noting each unique position we assembled an array of single photon detectors. Our scheme is illustrated in Fig. 3 (a) and (b) for the scalar and vector modes, respectively. 
The vector modes are mapped into ports $\mathrm{a}$ and $\mathrm{b}$ as following $\left|\phi_{k j}\right\rangle$,

$$
\left|\phi_{k j}\right\rangle \rightarrow \frac{1-e^{i \theta_{k j}}}{2}|\ell\rangle_{c}+i \frac{1+e^{i \theta_{k j}}}{2}|-\ell\rangle_{d},
$$

where $e^{i \theta_{k j}}$ is the intra-modal phase $\mathrm{e}^{30}$ that is resolved by the beam splitter. As a result each mode is detected unambiguously on output positions, $X_{\ell}$ resulting from the mode sorter transformation following

$$
X_{\ell}=\frac{\lambda f \ell}{d} .
$$

Here $\lambda$ is the wavelength, $\mathrm{f}=1000 \mathrm{~nm}$ is the focal length of the Fourier lens and $\mathrm{d}$ is the length of the unwrapped phase.

A detailed summary of the scalar and vector detection scheme can be found in ref. ${ }^{30}$ We measured the cross talk (scattering) between each mode set and present the results in Fig. 3 (c) and measured a fidelity of $\mathrm{F}=0.96$ with a corresponding error rate of $Q=0.04$. We measured the secure rate

$$
R=\log _{2}(d)+2 F \log _{2}(F)+2(1-F) \log _{2}\left(\frac{1-F}{d-1}\right),
$$

which was $R=1.39$ bits per photon, well above the theoretical bound of $R=1$ for the traditional implementation of the BB84 protocol with polarization states. We point out that deterministic sorting of the spatial modes is advantageous and outperforms sifting methods where each mode is detected probabilistically. ${ }^{30}$ Our scheme is the first to exploit high dimensionality of spatial modes with hybrid entangled (non-separable) states in high dimensions for quantum communication.

\section{CONCLUSION}

We have shown that hybrid entanglement can be exploited to realise new avenues of exploring the quantum eraser experiment. Moreover, we have shown that we can exploit spatial modes of light with a classical analogue of hybrid entanglement (vector modes) to demonstrate a high dimensional quantum key distribution protocol.

In addition we have recently exploited the quantum eraser scheme reported here with vector modes to characterize channel perturbations in a fiber medium. ${ }^{31}$ This will become invaluable for applications in quantum communication with hybrid entangled photon states and vector modes as an information basis. ${ }^{15,16,30,32,33}$

\section{REFERENCES}

[1] Aspect, A., Grangier, P., and Roger, G., "Experimental tests of realistic local theories via bell's theorem," Physical Review Letters 47(7), 460 (1981).

[2] Aspect, A., Grangier, P., and Roger, G., "Experimental realization of einstein-podolsky-rosen-bohm gedankenexperiment: a new violation of bell's inequalities," Physical Review Letters 49(2), 91 (1982).

[3] Kwiat, P. G., Waks, E., White, A. G., Appelbaum, I., and Eberhard, P. H., "Ultrabright source of polarization-entangled photons," Physical Review A 60(2), R773 (1999).

[4] Thew, R. T., Tanzilli, S., Tittel, W., Zbinden, H., and Gisin, N., "Experimental investigation of the robustness of partially entangled qubits over 11 km," Physical Review A 66(6), 062304 (2002).

[5] De Riedmatten, H., Marcikic, I., Scarani, V., Tittel, W., Zbinden, H., and Gisin, N., "Tailoring photonic entanglement in high-dimensional hilbert spaces," Physical Review A 69(5), 050304 (2004).

[6] Stucki, D., Zbinden, H., and Gisin, N., "A fabry-perot-like two-photon interferometer for high-dimensional time-bin entanglement," Journal of Modern Optics 52(18), 2637-2648 (2005).

[7] Mair, A., Vaziri, A., Weihs, G., and Zeilinger, A., "Entanglement of the orbital angular momentum states of photons," Nature 412(6844), 313-316 (2001).

[8] Dada, A. C., Leach, J., Buller, G. S., Padgett, M. J., and Andersson, E., "Experimental high-dimensional two-photon entanglement and violations of generalized bell inequalities," Nature Physics 7(9), 677-680 (2011). 
[9] Leach, J., Jack, B., Romero, J., Ritsch-Marte, M., Boyd, R., Jha, A., Barnett, S., Franke-Arnold, S., and Padgett, M., "Violation of a bell inequality in two-dimensional orbital angular momentum state-spaces," Optics express 17(10), 8287-8293 (2009).

[10] McLaren, M., Agnew, M., Leach, J., Roux, F. S., Padgett, M. J., Boyd, R. W., and Forbes, A., "Entangled bessel-gaussian beams," Optics Express 20(21), 23589-23597 (2012).

[11] Rossi, A., Vallone, G., Chiuri, A., De Martini, F., and Mataloni, P., "Multipath entanglement of two photons," Physical Review Letters 102(15), 153902 (2009).

[12] Barreiro, J. T., Wei, T.-C., and Kwiat, P. G., "Beating the channel capacity limit for linear photonic superdense coding," Nature Physics 4(4), 282-286 (2008).

[13] Neves, L., Lima, G., Delgado, A., and Saavedra, C., "Hybrid photonic entanglement: Realization, characterization, and applications," Physical Review A 80(4), 042322 (2009).

[14] Milione, G., Sztul, H., Nolan, D., and Alfano, R., "Higher-order poincaré sphere, stokes parameters, and the angular momentum of light," Physical Review Letters 107(5), 053601 (2011).

[15] Ndagano, B., Perez-Garcia, B., Roux, F. S., McLaren, M., Rosales-Guzman, C., Zhang, Y., Mouane, O., Hernandez-Aranda, R. I., Konrad, T., and Forbes, A., "Characterizing quantum channels with non-separable states of classical light," Nature Physics 13(4), 397-402 (2017).

[16] Milione, G., Lavery, M. P., Huang, H., Ren, Y., Xie, G., Nguyen, T. A., Karimi, E., Marrucci, L., Nolan, D. A., Alfano, R. R., et al., " $4 \times 20$ gbit/s mode division multiplexing over free space using vector modes and a q-plate mode (de) multiplexer," Optics Letters 40(9), 1980-1983 (2015).

[17] Souza, C., Borges, C., Khoury, a., Huguenin, J., Aolita, L., and Walborn, S., "Quantum key distribution without a shared reference frame," Physical Review A 77, 1-4 (2008).

[18] Vallone, G., DAmbrosio, V., Sponselli, A., Slussarenko, S., Marrucci, L., Sciarrino, F., and Villoresi, P., "Free-space quantum key distribution by rotation-invariant twisted photons," Physical Review Letters 113(6), 060503 (2014).

[19] Nagali, E. and Sciarrino, F., "Generation of hybrid polarization-orbital angular momentum entangled states," Optics express 18(17), 18243-18248 (2010).

[20] Karimi, E., Leach, J., Slussarenko, S., Piccirillo, B., Marrucci, L., Chen, L., She, W., Franke-Arnold, S., Padgett, M. J., and Santamato, E., "Spin-orbit hybrid entanglement of photons and quantum contextuality," Physical Review A 82(2), 022115 (2010).

[21] Bohr, N. et al., [The quantum postulate and the recent development of atomic theory], vol. 3, Printed in Great Britain by R. \& R. Clarke, Limited (1928).

[22] Franke-Arnold, S., Allen, L., and Padgett, M., "Advances in optical angular momentum," Laser 83 Photonics Reviews 2(4), 299-313 (2008).

[23] Nape, I., Ndagano, B., and Forbes, A., "Erasing the orbital angular momentum information of a photon," Physical Review A 95(5), 053859 (2017).

[24] Nagali, E., Sciarrino, F., De Martini, F., Marrucci, L., Piccirillo, B., Karimi, E., and Santamato, E., "Quantum information transfer from spin to orbital angular momentum of photons," Physical Review Letters 103(1), 013601 (2009).

[25] Walborn, S., Cunha, M. T., Pádua, S., and Monken, C., "Double-slit quantum eraser," Physical Review A 65(3), 033818 (2002).

[26] Lavery, M. P. J., Robertson, D. J., Sponselli, A., Courtial, J., Steinhoff, N. K., Tyler, G. A., Wilner, A. E., and Padgett, M. J., "Efficient measurement of an optical orbital-angular-momentum spectrum comprising more than 50 states," New Journal of Physics 15 (2013).

[27] Berkhout, G. C. G., Lavery, M. P. J., Courtial, J., Beijersbergen, M. W., and Padgett, M. J., "Efficient Sorting of Orbital Angular Momentum States of Light," Physical Review Letters 105, 153601 (oct 2010).

[28] Fickler, R., Lapkiewicz, R., Huber, M., Lavery, M. P., Padgett, M. J., and Zeilinger, A., "Interface between path and orbital angular momentum entanglement for high-dimensional photonic quantum information," Nature Communications 5, 5 (2014).

[29] Dudley, A., Mhlanga, T., Lavery, M. P. J., McDonald, A., Roux, F. S., Padgett, M., and Forbes, A., "Efficient sorting of Bessel beams.," Optics Express 21(1), 165-71 (2013). 
[30] Nape, I., Ndagano, B., Perez-Garcia, B., Scholes, S., Hernandez-Aranda, R. I., Konrad, T., and Forbes, A., "High-bit-rate quantum key distribution with entangled internal degrees of freedom of photons," arXiv preprint arXiv:1612.09261 (2016).

[31] Nape, I., Kyeremah, C., Vallés, A., Rosales-Guzmán, C., Buah-Bassuah, P. K., and Forbes, A., "A hybrid quantum eraser scheme for characterization of free-space and fiber communication channels," Optics Communications (2017).

[32] Cox, M. A., Rosales-Guzmán, C., Lavery, M. P. J., Versfeld, D. J., and Forbes, A., "On the resilience of scalar and vector vortex modes in turbulence," Optics Express 24(16), 18105 (2016).

[33] Ndagano, B., Brüning, R., McLaren, M., Duparré, M., and Forbes, A., "Fiber propagation of vector modes," Optics Express 23, 17330 (jun 2015). 